patients have recovered with no special treatment when placed in even the imperfect isolation obtainable in an ordinary hospital ward. In the Salpêtrière Professor Dejerine has secured what proves to be a really sufficient degree of isolation by giving up to neurasthenic and hysterical patients one whole ward, in which the beds are screened off from each other.

We must not, however, hastily assume that isolation is all that is generally needed. No, if the attempt to analyse the neurasthenic condition that has just been made is in any degree correct, clearly what has to be aimed at is to reverse the narrowing process; first to get rid of the harassing notion of diseased organs; then to leave behind the over-insistent ideas of personal trouble and distress that have held sway; to draw up the darkening blinds, let daylight in and look further afield upon life ; to cultivate a self of larger scope. We may surmise that the special virtue of isolation in unwonted surroundings lies in the fact that nothing else so quickly and completely breaks the domination of habitual ideas and makes way for others furnishing a better adjustment to life. Of course, physical means will not be neglected in cases where protracted nervous illness has rendered them necessary or advisable: rest in bed (reduced as the patient recovers strength and vitality), overfeeding in cases of emaciation, exercise of the muscles by massage and electricity - all these will have their value recognized as useful accessories; but systematic treatment should be elastic and differently adapted to meet the needs of different temperaments and circumstances in patients, no two of whom. are ever quite alike. What we want to secure is a central will, reassured, instructed, strengthened, and set free from worrying trammels to play its proper part as director-general of the personality.

It must be added that the chances of treating neurasthenic cases successfully as part of an ordinary general practice are not very good. The need for treating them away from home and with the aid of carefully chosen nurses, the objections to routine treatment, above all the superlative importance of obtaining the patient's entire confidence, and the incalculable influence of the physician's personality in this respect-all point to the ad vantages of specialist treatment for this kind of illness. Constant experience is as valuable to the physician in dealing with neurosis as constant practice is to the surgeon who undertakes the more critical kind of operations. Tact, sympathy, firmness, resource, and infinite patience are called for and must be kept bright with use Therefore the practitioner who does not find in himself any particular aptitude or interest in dealing with neurotic patients would be well advised to commend them to a specialist; on the other hand, a doctor who discovers that such patients will spontaneously confide in him and confess private troubles and even faults, in their longing for advice and relief, has a large sphere of helpfulness open to him if he chooses to take possession.

\section{ON CANCER.}

By SIR SAMUEL WILKS, BarT., M.D., F.R.S.

As I have always been much interested in the subject of cancer, I read the British Medical Journal of May '26th, giving an account of the work done by the Cancer Research Fund, with especial pleasure, and also your leader containing such an admirable summary of the present state of our knowledge and the problems for the future. My pleasure was naturally increased by the personal satisfaction which I experienced on seeing that the conclusions arrived at were so closely allied to the doctrines which I had for many years taught in my own school. These doctrines were not vague opinions arising from a non-acceptance of the prevailing views, nor hypothetical ones invented to take their place, but substantial and legitimate conclusions based on a large number of facts and on a long study of the subject. The views which I expressed were first published in the Guy's Hospital Reports for the year 1857-nearly half a century ago-in a paper on New Growths from Bone, and this was developed into a longer one in the next number of the Reports on Cancer and Malignancy.

At that time the microscope was coming into use and its value was considered very great, by enabling us to distinguish between malignant and benign growths. This proved to be true, as it rendered it possible to see in any particular growth a mass of cells and in another nothing but fibres. The first was cancer and malignant, being associated with all the clinical facts of the disease, whilst the other was innocent. These cells were supposed to be typical of cancer, being large, rounded cells with a large oval nucleus, and contained in a matrix. These characteristics combined with the history of the case were sufficient to decide its cancerous nature. It was a different question, however, to ascertain whether these cells showed any peculiarity of form or structure, and so after spending much time at this work, I came to the conclusion that they showed no special characters which could differentiate them as cancer cells from all others; I was thus placed in opposition to the current opinion of the day. I believed I had satisfactorily proved my position, for when I placed some healthy tissues under the microscope, and more particularly lymph taken from inflamed joints, and submitted them to competent observers as new growths, they were generally pronounced to be cancer if they showed a large nuclear cell. This was always the case if I gave any suggestion as to possible malignancy.

I came, therefore, to the conclusion that cancer cells had no characteristics distinctive enough to allow them to be marked off from others; indeed, they were no more in most instances than the ordinary embryonic cells. The belief at that time was that cancer was the outward and visible sign of a malignant disease arising from some unknown cause in the system. This seemed to be very evident when the disease returned after it had been removed; if it did not do so it showed that the original growth was not cancer. So there was a good deal of circular reasoning on the subject.

It was thought also that there was a malignant aspect due to a special kind of cachexia. This illusory notion I got rid of whilst a student, for I soon saw a middle-aged, healthy-looking woman apply at the hospital in consequence of her having found, whilst dressing that morning, a lump in her breast. This the assistant surgeon pronounced to be scirrhus. And then again, when an incident occurred of an opposite kind. This was the case of a man with long-standing jaundice, which at last had made his face dark green, or almost black. The very experienced physician, although he could find no tumour in the abdomen, pronounced the case to be undoubted cancer owing to the patient's malignant appearance and his great emaciation. After his death no trace of cancer could be found, but the common bile duct was obliterated in consequence apparently of ulceration caused by a gall stone.

I should say that the physiological doctrine of growth then taught was that of Schwann and Schleiden. It was that the blood nourished the body by exuding a fluid which was called the blastema; in this fluid granules shortly appeared; these subsequently formed themselves into nuclei, and around them the cell-wall grew. Out of this cytoblastema all the tissues of the body arose or were regenerated, the cell being converted in one case into a nerve fibre and in another case into a muscular fibre, and so on with all the tissues. My theory of cancer was that if these cells did not do their duty and follow their destination so as to produce natural tissue, but convinued to increase in number without developing, they formed a mass or tumour, and to this the name of cancer was given. They corresponded in form to the cells of malignant growths, and therefore the latter were nothing more than masses of embryonic cells. It was a purposeless growth, and therefore might in contradistinction to benignant correctly be styled malignant. The cells were usually contained in a newly-formed matrix with a good supply of blood vessels. If the new material showed any attempt to grow, then the cell might become elongated until a simple fibre was formed. In this way I made a scale beginning with the cancer or simple cell growth, and so passing on with its development until I reached the simplest and most innocent pure fibrous tumour.

In my papers I discussed another important feature of new growths which made them totally unlike what is seen in specific diseases, and that is that they differed according to the part of the body in which they grew. In the case of any ordinary well-known virus, as that of measles or small-pox, or even that of tubercle, the effects are uniform 
as seen especially on the mucous membranes and skin; but the so-called cancer if growing on a bone might contain osseous matter, cartilage or a myeloid material, and if it sprung from the choroid of the eye would be melanotic ; moreover, all these local characters might be propagated to the lungs and other parts. Even a local adenoid growth might be found as a secondary formation in a distant part, carrying all its definite peculiarities with it. Besides, therefore, the absence of any special characters in the cancer cell, we had to deal with the fact that unlike tubercle it altered its characters in all parts of the body in which it occurred. These facts always made me in later years sceptical when I heard of any specific germ having been found, and of necessity the more so when it was found not to be infective.

I will now give one or two extracts from the papers I mention in which the terms heterologous and analogous were given by Carswell to growths consisting of elements foreign to the system and those which resembled them. Such a division has been more or less accepted up to the present time. 'The following is from my first paper written in 1857 :

The question as to the malignancy of a new growth is the most important which can be asked concerning it, for, should the answer be in the affirmative, the decision is almost equivalent to the passing judgement of a certain and speedy death upon the unfortunate subject of it. The importance, therefore, is a sufficient excuse for making a few remarks upon the terms cancer and malignant disease. Cancer, although divided into several varieties, has generally been regarded as a disease consisting of a specific morbid material, which, once having appeared in any part of the system, resists all attempts at eradication or cure, since, as often as removed, it returns, or, having gained strength by age, at last breaks forth with virulence, until several distinct regions of the body have yielded to its destructive influence. Such a malady is eminently malignant, and thus the words cancer and malignant disease have grown up side by side as synonymous terms, the one having reference to the anatomical nature of the growth, and the other to its widespread ruinous character. When, therefore, in times past a new growth was early perceived, the importance of anew growth was early perceived, the importance of this frightful course of malignancy was reasonable. At so incipient a stage the naked-eye appearance would not solve the question, and recourse was had to the microscope to assist the surgeon in his inquiry, for great expectations were then formed of the value of this instrument in respect to its ability to determine the cancerous or non-cancerous nature of a growth and thus form a decision upon its malignature of a growth and thus form a decision upon its malignancy. Much disappointment, however, has been the result of
this hope and discredit thrown on the means emp'oyed, but this hope and discredit thrown on the means emp'oyed, but this has been without good reason, for we believe that the ingation, and that its supposed failure has been in reality a bright eature in favour of its utility and importance. Its disparagement has been due not so much to its occasional indecisive answers as to the fact of its denial of the cancerous nature of many growths which returned after removal or even multiplied themselves in various organs of the body with the utmost degree of malignancy. The microscope has therefore proved that cancer and malignant disease are not universally interchangeable terms, and all praise rather than blame is due e instrument for the discovery.

The following is from a second paper on the question of local or constitutional causes:

To discover how much one cause is in operation, and how much the other, we think is the clue to the whole question of new growths, and in this we see a meaning in the terms new growths, and in this we see a meaning in the terms if the latter be allied to the structure near which they spring, if they indicate a healthy influence in operation, and therefore an absence of any vicious constitutional tendency, whereas, if they be not allied, that is consist of cells continually growing for a purposeless object, they indicate a vice in the system the latter is foreign to a healthy intent, and therefore deserves the name heterologous. In the one case the tendency is to a formative process, and the nearer this approaches to the natural one the more benignant the structure. while in the other case the tendency is to the production of cells such as existed in the embryo preceding the formation of the tissue, and therefore if such embryonic cells spring up and continue to grow in the perfected body we have a cancer, a cancerous tumour being simply a mass of embryonic cells.

These quotations contain an outline of the views which I put forth half a century ago on the nature of cancer in the Guy's Hospital Reports. It is naturally of much interest to myself to see how the tendency of the work which is now being carried on has so much agreement with that which I had formerly written. Were this only a personal satisfaction I should not have dared to encroach so much on your space, but I regard it as of wider value, and especially to those who are now engaged in the cancer research. For it must be remembered that all our knowledge of natural phenomena can only be partial and relative; therefore it follows that having been gained step by step, we ought to be well acquainted with all the graduations which preceded it up to the present time. I have long been convinced that a pupil gains a more thorough grasp of any subject in natural philosophy if the lecturer begins to speak of the very first faint observation and then proceeds onwards until he reaches the present state of knowledge.

With this view I think it useful to know in what position we stood in connexion with cancer and allied growths when the microscope first began to be used for its investigation. This was the purport of my papers which I mention. In these I say that this instrument was of much use in showing the nature of cancer growth in distinction to that which is innocent, but it failed to prove that it had any characteristics which could at once separate it from normal structures. These views were not incompatible with the belief that, if the disease is one of nutrition, the cause might be of a special character although morbid; in other words I believed that the disease had its origin in the body itself, and was not of external origin. This idea was afterwards strengthened by observing the dire effects on nutrition when disease occurred in the thyroid, suprarenal, and perhaps the pituitary body. As regards the future attainment of the knowledge we require, we ought not to be over-sanguine as to its now being within our reach, as perhaps some altogether new method may be necessary to get a further insight into it. As it is now many years since Müller employed all the well-known chemical methods to discover the difference between a natural and malignant growth, so it may be that we require a new biochemistry to solve the problem. When the microscope came into use it enabled us to trace the structure of organic bcdies imperceivable by the naked eye, and actually to see the poisonous matter, which we only before conjectured to be present in the ordinary specific diseases. The importance of new methods of investigation has been shown by the great advance of medicine owing to the introduction of the laryngoscope, ophthalmoscope, and other instruments. Without them we should have been only slightly better than our forefathers, having only our ordinary senses to guide us. In all branches of knowledge it is the same, new methods and instruments of investigation are necessary. The study of the heavens was complete as far as the telescope could make it, when astronomy made a quick advance by the aid of photography and the spectroscope. It may be, therefore, that we require some new methods of research before we can alight upon the true nature of cancer; but let us hope that the desideratum may not be far off, for every one must have been struck by the remarkable advances made in a knowledge of some of the most intricate functions of the body by our present distinguished physiologists.

UNDER the will of the late Dr. Thomas Corbett of Droitwich, who died on April 22 nd, the following sums are allotted to the institutions undermentioned: To St. Thomas's Hospital, for the endowment of beds in memory of the deceased's brother. £5.C00, and £5C0 to its Samaritan Fund; to Guy's, the Middlesex, St. George's, University College Hospital. the East London Hospital for Children, and the Hospital for Sick Children, Great Ormond Street, $£ 5,000$ each; to the Royal Free, Gray's Inn Road, the General Hospital, Birmingham, and the Queen's Hospital in the same town, mingham, and to the Cancer Hospital. Fulham, Gloucester Infirmary, Royal Hospital for Incurables, Putney, Belgrave Hospital for Children, Pimlico, and the Worcester Infirmary, $£ 2,000$ each, the latter institution receiving, also, a further additional bequest of $£ 500$ : to the Rhyl Confurther add the Seaford Convalescent Home, the Midland Home for Incurables, Leamington, the Alexandra Hospital for Hip Disease in Children, the British Medical Benevolent Fund, and the Royal Medical Benevolent College, Epsom, $£ 1,000$ each. Directions are included in the will for the perpetual provision of medical attendance on the occupants of some almshouses built by his brother at an expense of £25 a year. 